\title{
Atuação do Psicólogo na Gestão Integral de Riscos e Desastres: Uma Revisão Sistemática da Literatura
}

\section{Psychologist's Practices in Integral Management of Risks and Disasters: A Systematic Literature Review}

\author{
Marina Padilha Ribeiro (orcid.org/0000-0002-2574-2460) ${ }^{1}$ \\ Joanneliese de Lucas Freitas (orcid.org/0000-0002-0856-3460)²
}

\begin{abstract}
Resumo
Desastre é um conceito complexo, envolvendo fatores sociológicos e psicológicos. Sua compreensão inclui a gestão dos riscos e o manejo da relação de diferentes grupos com suas vulnerabilidades. Estudos sobre a atuação dos psicólogos na gestão integral de riscos e desastres são recentes no Brasil. Foi realizada uma revisão sistemática qualitativa que visou mapear na literatura acadêmica orientações para a atuação de psicólogos na área. Foram analisados 14 trabalhos utilizando-se a técnica de leitura e de classificação em tabela por meio do protocolo Prisma. Nota-se que não há consenso sobre a atuação do psicólogo e que as orientações estão concentradas no pós-desastre, quando surge relevante debate sobre a aplicabilidade do debriefing psicológico. Concluiu-se que é necessário ampliar os estudos e a participação do psicólogo na gestão de riscos e na prevenção, além da sua inclusão em equipes que atuem não apenas nos desastres, mas também na gestão pública.
\end{abstract}

Palavras-chave: Desastres. Emergências. Riscos. Psicologia.

\begin{abstract}
Disaster is a complex concept concerning sociological and psychological factors. Its understanding includes risk management and managing the relationship of different groups with their vulnerabilities. Studies on the psychologists' practice on risk and disaster management are recent in Brazil. A systematic qualitative review was conducted to outline the academic literature about the guidelines for psychologists in the field. A total of 14 studies were analyzed using the technique of reading and table classification using the PRISMA protocol. It is noted that there is no consensus on the psychologist's practice and that the guidelines are focused on post-disaster when there is a relevant debate on the psychological debriefing's applicability. It was concluded that it is necessary to expand the studies and improve the psychologist's participation in risk management and prevention withal her inclusion in teams that work not only in disaster management but also in public management.
\end{abstract}

Keywords: Disasters. Emergencies. Risks. Psychology.

\footnotetext{
${ }^{1}$ Universidade Federal do Paraná, Curitiba, Brasil. E-mail: ma.ilha1910@gmail.com.

${ }^{2}$ Universidade Federal do Paraná, Curitiba, Brasil. E-mail: joanneliese@gmail.com.
} 


\section{Introdução}

Indesejados e temidos, os desastres podem ou não acontecer por influência do ser humano. Tendo em vista o aumento da população e a falta de informação e cuidados com a natureza, a tendência é que acidentes ocorram cada vez mais e com maior frequência. Apesar de muitas vezes não serem tão valorizados quanto os cuidados físicos e os prejuízos materiais, os cuidados psicológicos antes, durante e depois dos desastres são de extrema relevância para que as pessoas afetadas possam reorganizar suas vidas e manter a saúde mental.

De acordo com a Organização PanAmericana da Saúde (Opas, 2014, p. 9), um desastre ocorre quando há "uma séria interrupção do funcionamento normal de uma comunidade ou sociedade, afetando seu cotidiano", excedendo a sua capacidade em lidar com a situação com seus próprios recursos, necessitando de auxílio externo. Tal interrupção é constituída por perdas que podem ser de todos os tipos, desde materiais até humanas. Segundo Franco (2015), a Cruz Vermelha Internacional (CVI) divide os desastres em dois tipos: naturais, por exemplo, terremotos, tempestades e deslizamentos de terra, e tecnológicos, como os acidentes industriais e os com transportes. As emergências, por sua vez, são as situações inesperadas e anormais, provocadas pelos desastres, que causam prejuízos de diversos tipos para a sociedade, porém, neste caso, segundo o conceito da Secretaria Nacional de Defesa Civil (SNDC, 2017), os danos implicam no comprometimento apenas parcial de resposta do poder público.

O Centro Universitário de Estudos e Pesquisas sobre Desastres (Ceped) da Universidade Federal de Santa Catarina (UFSC) em parceria com a SNDC (Ceped UFSC, 2013), realizou um estudo para levantar dados estatísticos a respeito das ocorrências de desastres naturais no Brasil entre os anos 1991 e 2012. Entre os desastres elencados, encontramse estiagem e seca, inundação brusca e alagamento, inundação gradual, vendaval ou ciclone, tornado, granizo, geada, incêndio florestal, movimento de massa, erosão fluvial, erosão linear e erosão marinha. A pesquisa constatou um aumento de $78 \%$ nos desastres nos últimos 13 anos do estudo (1991-2012). Segundo o Ceped UFSC (2013), tal informação pode estar comprometida, visto que o sistema da Defesa Civil apresenta dificuldades em manter atualizados seus registros. Porém, mesmo que tais dados não reflitam a totalidade das ocorrências, nota-se que o aumento dos desastres é alto e preocupante.

A literatura nacional sobre a atuação do psicólogo na gestão integral de riscos e de desastres conta atualmente com três obras. $\mathrm{O}$ primeiro livro publicado sobre o tema, Gestão de riscos e de desastres: contribuiçoes da Psicologia (Ceped UFSC, 2010), trata do material de um curso a distância realizado pelo Ceped UFSC, que teve como finalidade apresentar e divulgar o trabalho do psicólogo no contexto da gestão de risco e de desastres. O livro Intervenção psicológica em emergências: fundamentos para a prática (Franco, 2015) se constitui por uma coletânea de artigos escritos por diversos profissionais com experiência na área, organizado pela Profa. Dra. 
Maria Helena Pereira Franco. A terceira obra, recentemente lançada pela editora Hogrefe, $O$ psicólogo na redução dos riscos e desastres, é uma coletânea de artigos organizada por Lopes e Filho (2017), que trata do papel do psicólogo na redução de riscos e de desastres.

No que se refere às definições sobre desastres, Favero, Sarriera e Trindade (2014) realizaram uma revisão da literatura com o objetivo de discutir os conceitos a partir das perspectivas da Sociologia e da Psicologia. Favero et al. (2014) afirmam que definir "desastre" é uma tarefa complexa, que deve ser compreendida levando em consideração diversos fatores dos contextos físico, social, político e econômico. Os autores defendem que os conceitos utilizados devem ser pautados nas áreas de estudo da Psicologia e da Sociologia, posto que as organizações que atuam em situações de desastres, tais como SNDC, Opas e CVI, utilizam critérios aplicados de acordo com interesses próprios. Assim, Favero et al. (2014) propõem um conceito amplo que inclui diferentes aspectos apontados por diversos autores, integrando-os em um único conceito, no qual se articulam aspectos sociológicos e psicológicos. Para os autores, desastre se caracteriza como

um processo que tem sua origem na interação entre seres humanos e seu contexto social (Britton, 1986), salientando-se que, mais do que um evento agudo, um desastre é a expressão aguda da vulnerabilidade em suas diferentes dimensões (física, social, ambiental, etc.). Os desastres desafiam a capacidade humana de resposta (Quarantelli, 1985), podendo trazer consigo perdas (Fritz, 1961) repentinas e prolongadas no tempo. Eles podem ser cíclicos ou escalonados, de início súbito e com grande potencial traumático (Garcia-Renedo et al., 2007; McFarlane \& Norris, 2006); ou ainda, podem se desenvolver de maneira lenta, de modo a expor indivíduos e grupos a prolongados períodos de estresse (Favero, Sarriera, Trindade, \& Galli, 2013). (Favero et al., 2014, p. 207)

Os desastres podem ser divididos em momentos distintos: o anterior, ou pré-desastre; o momento durante o desastre; e o posterior, ou pós-desastre. O profissional de Psicologia pode atuar em todos eles, seja no treinamento de uma equipe de socorristas para que saibam acolher as vítimas com os primeiros cuidados psicológicos, seja realizando projetos de conscientização da população e criação de vínculos com a rede de serviços local, ou até mesmo trabalhando diretamente nos locais da tragédia com as vítimas e seus familiares.

No tocante à população acolhida, apesar de a regulamentação governamental sobre assistência às famílias de passageiros vitimados em desastres aéreos do Departamento de Aviação Civil (DAC) ditar que os serviços em Psicologia devam ser oferecidos apenas às famílias das vítimas, Franco (2005) instrui que os atendimentos sejam realizados também com funcionários da empresa, moradores das ruas atingidas, ou qualquer outra pessoa que seja afetada pelo desastre, estejam feridos ou não. 
Por mais que existam inúmeras formas de atuação do psicólogo na gestão integral de riscos e desastres, os alunos de graduação em Psicologia saem da academia com pouca ou mesmo sem qualquer orientação ou preparo para agir em tais contextos. Depois de breve análise das grades curriculares de 36 universidades federais e estaduais brasileiras, ${ }^{3}$ constatou-se que apenas três delas oferecem disciplinas que poderiam tratar - de forma aproximada - sobre a atuação do psicólogo nesses contextos. Destas, todas são optativas, a saber: "Intervenção em crise", na Universidade

3 Foram consultados os sites das seguintes Instituições Federais: Universidade de Brasília (UnB), Universidade Federal de Alagoas (Ufal), Universidade Federal da Bahia (UFBA), Universidade Federal do Ceará (UFC), Universidade Federal do Espírito Santo (Ufes), Universidade Federal Fluminense (UFF), Universidade Federal de Goiás (UFG), Universidade Federal de Juiz de Fora (UFJF), Universidade Federal do Maranhão (UFMA), Universidade Federal do Mato Grosso (UFMT), Universidade Federal de Minas Gerais (UFMG), Universidade Federal do Pará (UFPA), Universidade Federal da Paraíba (UFPB), Universidade Federal do Paraná (UFPR), Universidade Federal de Pelotas (UFPel), Universidade Federal do Piaú (UFPI), Universidade Federal do Rio Grande do Norte (UFRN), Universidade Federal do Rio Grande do Sul (UFRGS), Universidade Federal do Rio de Janeiro (UFRJ), Universidade Federal de Santa Catarina (UFSC), Universidade Federal de Santa Maria (UFSM), Universidade Federal de São Carlos (UFSCar), Universidade Federal de São Paulo (Unifesp), Universidade Federal de Sergipe (UFS), Universidade Federal do Triângulo Mineiro (UFTM), Universidade Federal de Uberlândia (UFU). E as seguintes Instituições Estaduais: Universidade do Estado da Bahia (Uneb), Universidade Estadual do Ceará (Uece), Universidade Estadual do Centro-Oeste (Unicentro), Universidade Estadual de Londrina (UEL), Universidade Estadual de Maringá (UEM), Universidade Estadual da Paraíba (UEPB), Universidade Estadual Paulista (Unesp), Universidade Estadual do Piaú (Uespi), Universidade Estadual do Rio de Janeiro (Uerj), Universidade de São Paulo (USP). de Brasília (UnB), "Terapia breve e de emergência", na Universidade Federal de Minas Gerais (UFMG) e "Intervenções psicoterápicas em situações de crise", na Universidade Federal do Maranhão (UFMA). Soma-se isso ao fato de os profissionais de outras áreas que atuam diretamente nas catástrofes também não receberem treinamento referente aos Primeiros Cuidados Psicológicos (PCP), intervenção fundamentada nas diretrizes da Organização Mundial da Saúde - OMS (2015).

Apesar de o Conselho Federal de Psicologia (CFP) ter produzido duas Notas Técnicas (2013 e 2016) a respeito da atuação do psicólogo nesses contextos e constar no Código de Ética (CFP, 2005) da profissão (em seu art. $1^{\circ}$ al. d) que é dever do Psicólogo "prestar serviços profissionais em situações de calamidade pública ou de emergência, sem visar benefício pessoal" (p. 8), chama atenção que hoje não exista no Brasil um protocolo de atendimento com orientações para psicólogos sobre intervenção em emergências e desastres.

Em uma revisão da literatura sobre a atuação do psicólogo em desastres naturais, abrangendo o período de 2000 a 2010, Alves, Lacerda e Legal (2012) encontraram 30 trabalhos sobre o tema. A partir desse estudo, os autores constataram que o Brasil tem poucas publicações acadêmicas sobre a intervenção do psicólogo em desastres naturais. Além disso, afirmam que as publicações são de difícil acesso, visto que os descritores "Psicologia" e "desastres", "desastres naturais" e "Psicologia dos desastres", utilizados pelos autores durante a pesquisa, não os conduziram aos trabalhos sobre tais temas; desse modo, julgaram necessário 
buscá-los a partir das referências bibliográficas dos resultados encontrados. No que se refere especificamente à prática do psicólogo, a maioria das pesquisas incluídas na revisão de Alves et al. é focada na atuação pós-desastre. Portanto, concluem os autores, fazem-se necessários mais estudos sobre as ações de prevenção anteriores aos desastres.

Segundo o Ceped UFSC (2010), a denominação recomendada quando se fala sobre a atuação do psicólogo em emergências e desastres é "Psicologia da gestão de riscos e de desastres", cuja denominação diz respeito a um trabalho que engloba a construção de políticas públicas e práticas de prevenção (Ceped UFSC, 2010, p. 19). Em 2016, o CFP modificou o nome do campo para "Psicologia na gestão integral de riscos e desastres" (CFP, 2016).

Diante das dificuldades de definição do que é um desastre, também há a dificuldade de encontrarmos orientações e diretivas para o trabalho e atuação do psicólogo na gestão integral de riscos e de desastres. Nesse sentido, nos perguntamos quais seriam as orientações para a atuação do psicólogo nesses contextos, diante da ausência de protocolos no Brasil e como os trabalhos acadêmicos podem contribuir para lidar com essa falta. O presente estudo trata-se de uma revisão de literatura que teve como finalidade mapear o que existe de orientações para a atuação dos psicólogos no contexto da gestão integral de riscos e desastres no Brasil. Tal mapeamento permite entender e localizar quando o estudo sobre a atuação do psicólogo em tais contextos teve início no campo acadêmico brasileiro, a evolução das publicações e suas sazonalidades, quais são seus tipos e os métodos, auxiliando a encontrar avanços e também lacunas.

\section{Método}

Foi realizada uma revisão sistemática qualitativa em busca da descrição de orientações existentes para a atuação do psicólogo na gestão de riscos e desastres na literatura acadêmica brasileira. EM 21 de fevereiro de 2017, foram realizadas todas as buscas descritas no trabalho com as seguintes palavras-chave: "Psicologia das emergências e desastres", "primeiros socorros psicológicos" e "intervenção psicológica nas emergências e desastres".

Em um primeiro momento, foram realizadas buscas nas seguintes bases de dados: Coordenação de Aperfeiçoamento de Pessoal de Nível Superior (Capes), Scientific Electronic Library Online (SciELO) e Periódicos Eletrônicos de Psicologia (PePSIC). Devido ao pequeno número de resultados, foi realizada uma segunda busca no portal do Google Acadêmico, no mesmo dia.

A análise dos trabalhos encontrados foi realizada utilizando a técnica de leitura e de classificação em tabela por meio do instrumento de checklist do Protocolo Prisma (Moher et al., 2015), estruturado basicamente em três etapas, quais sejam, identificação, seleção e elegibilidade. A etapa de identificação visou reunir e identificar todos os artigos encontrados a partir das palavras-chave supracitadas. Ela foi especialmente importante para classificação dos resultados obtidos no Google Acadêmico, que disponibilizou publicações que continham os termos da busca no texto, mas não 
necessariamente como palavras-chave da publicação. Os trabalhos deveriam necessariamente apontar para orientações sobre a atuação do psicólogo na gestão de risco e desastres. Destaca-se que não foi feita nenhuma restrição terminológica que diminuiria muito o número de trabalhos incluídos, ou seja, mesmo que se utilizassem terminologias diversas, os artigos foram considerados. $\mathrm{Na}$ etapa da seleção, foi aplicada a técnica de leitura e classificação em tabela por meio do checklist do Protocolo Prisma (Moher et al., 2015). Nesta etapa, foram excluídos os trabalhos repetidos e os que não continham texto completo. $\mathrm{Na}$ etapa da elegibilidade, aplicaram-se os critérios de inclusão e exclusão com a finalidade de serem separados os artigos elegíveis para serem estudados e avaliados, por meio da leitura dos resumos.

Os critérios de inclusão foram: 1 . Temático: todos os trabalhos acadêmicos completos que abordassem a atuação do psicólogo na gestão de riscos e de desastres foram incluídos, a saber, monografias, dissertações, teses e artigos; e 2. Trabalhos publicados no Brasil, em língua portuguesa, pois nos interessava compreender o contexto brasileiro, considerando os resultados de Alves et al. (2012), que apontam baixa produção sobre o tema no país. Os critérios de exclusão foram: 1. Artigos sem referência completa; e 2. Artigos que não foram publicados em periódicos científicos revisados por pares. Não houve exclusão por data de publicação.

Os trabalhos resultantes foram lidos integralmente e organizados em uma tabela, na qual foram destacados os tópicos principais e pontos de similaridade e discordância. Foram avaliados especialmente os seguintes tópicos: período de publicação (ano), com a finalidade de descrever desde quando têm sido realizadas publicações sobre o tema e suas sazonalidades, os tipos de trabalho e métodos utilizados e, por fim, quais as intervenções propostas. Sobre as intervenções propostas, foi realizada uma análise qualitativa mais cuidadosa, em busca de uma reflexão sobre divergências e convergências que possam caracterizar como no Brasil entende-se o trabalho do psicólogo na gestão de riscos e desastres. Essas intervenções foram analisadas a partir de três tempos discriminados: antes, durante e depois do desastre.

\section{Resultados e Discussão}

A etapa de identificação apresentou dois momentos: a busca nas bases de dados PePSIC, SciELO e Portal Capes e a busca no Google Acadêmico. Não houve quaisquer resultados na PePSIC. Na SciELO, foi encontrado apenas um artigo, repetido quando utilizadas as palavraschave "Psicologia das emergências e desastres" e “intervenção psicológica nas emergências e desastres". Já na plataforma Capes, foram encontrados 31 artigos no total, sendo $11 \mathrm{em}$ "Psicologia das emergências e desastres", 16 em "primeiros socorros psicológicos" e quatro em “intervenção psicológica em emergências e desastres". A soma dos artigos encontrados nessas bases de dados foi de 33. Como explicitado no método, foi também realizada uma pesquisa com as mesmas palavras-chave no Google Acadêmico, resultando em 31.960 trabalhos, que depois de aplicados os critérios de 
identificação, conforme descrito no método, restou um total de 39 trabalhos. A etapa de identificação totalizou, ao final, 72 itens, sendo 33 resultantes da primeira busca (PePSIC, SciELO e Portal Capes) e 39 da busca no Google Acadêmico.

$\mathrm{Na}$ etapa de seleção, foram excluídos 29 itens, que eram repetidos ou estavam sem texto completo, restando 43 trabalhos rastreados. Por fim, na etapa da elegibilidade, depois de aplicados os critérios de inclusão e exclusão, o número de trabalhos restantes foi de 14, dos quais temos 11 artigos, duas monografias e uma dissertação.

Assim como ocorreu no estudo de Alves et al. (2012), os descritores foram uma barreira para a execução da busca bibliográfica, pois por meio deles foram alcançados poucos resultados. A maioria das publicações utilizadas neste artigo foi encontrada no Google Acadêmico $(n=10)$. Embora este estudo tenha a mesma finalidade do estudo de Alves et al., observou-se que a maioria dos trabalhos nacionais encontrados é diferente. Deve-se considerar que algumas revistas não aceitam palavras-chave que não constem no dicionário de terminologia da Biblioteca Virtual em Saúde (BVS-psi), tais como a palavra "desastres" e "gestão de riscos". Desse modo, torna-se difícil localizar trabalhos que, mesmo tratando do tema, não são contemplados nas terminologias que constam nesse dicionário e acabam se utilizando de palavras-chave diversas.

\section{Ano de publicação}

Conforme demonstrado na Figura 1, o primeiro trabalho sobre a atuação do psicólogo na gestão integral de riscos e desastres é de 2005 , sendo que as publicações apresentam pico em 2013. Não há trabalhos depois de 2016, provavelmente em função da data da busca.

Figura 1. Quantidade de materiais de acordo com o ano de publicação

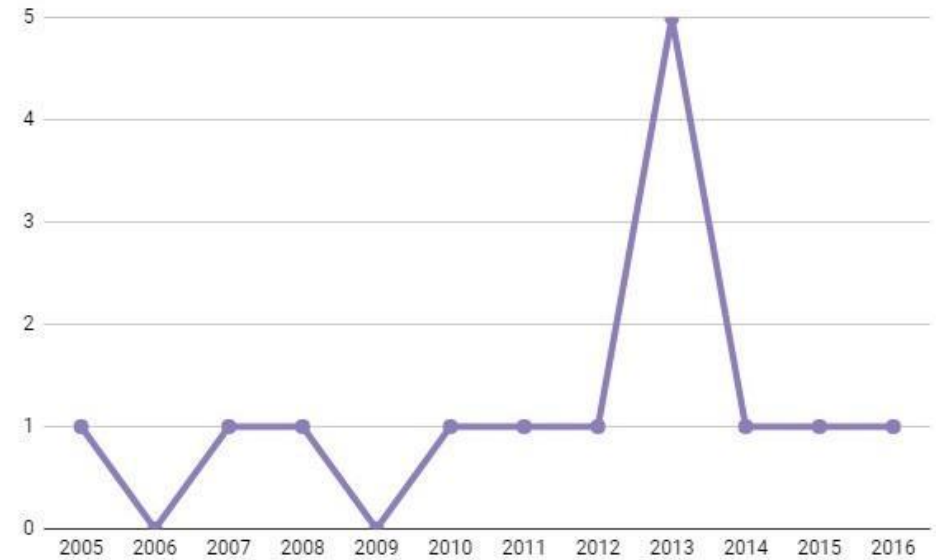

Fonte: Elaborada pelas autoras.

O estudo sobre gestão integral de riscos e de desastres é ainda recente no Brasil, visto que o artigo mais antigo encontrado é do ano de
2005. Na revisão de literatura realizada por Alves et al. (2012), entre os anos 2000 e 2010, foram encontrados apenas quatro trabalhos em 
língua portuguesa, entre os quais o mais antigo também é de 2005 e os três mais recentes são de 2008.

No intervalo apresentado, conforme Figura 1, observa-se que houve uma média de uma publicação por ano, que pode ser considerada baixa, especialmente se se levar em consideração a incidência de desastres que ocorrem no país, como os deslizamentos na região Serrana do Rio de Janeiro, as secas no Nordeste e as inundações em Santa Catarina, o que demanda certamente a atuação de psicólogos em parceria com outros profissionais. De acordo com o levantamento realizado pelo Ceped UFSC (2013), o número dos desastres naturais registrados no Brasil no período entre 2004 e 2012 foi de 23.180, apontando para uma discrepância entre a quantidade de desastres no país e o número de estudos acadêmicos sobre o tema, especialmente no que concerne à atuação do profissional de Psicologia.

Chama atenção o fato de o ano de 2013 ter apresentado pico, com um total de cinco publicações. Esse fato pode ser explicado pelo fato de, no Brasil, aquele ano ter sido marcado pela tragédia do incêndio na Boate Kiss em Santa Maria/RS. Devido a diversos fatores que envolveram a tragédia, tais como o contexto do desastre, a faixa etária das vítimas e a quantidade de mortos, a repercussão dada pela mídia foi imensa, e acredita-se que o ocorrido tenha impulsionado os psicólogos a pensar mais sobre o seu trabalho diante de situações como essa. Outro aspecto relevante que pode ter influenciado nessa produção é o fato de que muitas vítimas eram estudantes da Universidade Federal de Santa Maria (UFSM), que se mobilizou em diversas frentes para oferecer suporte à comunidade atingida.

\section{Métodos de trabalho e tipos de produção}

Os trabalhos mapeados no presente estudo foram 11 artigos, duas monografias e uma dissertação $(n=14)$; salienta-se que não foram encontradas teses de doutorado, assim como também não se constatou o predomínio de uma linha teórica da Psicologia nos trabalhos. Verifica-se como o tema da atuação do psicólogo na gestão de riscos e desastres é ainda pouco estudado no Brasil, visto que, além de não terem sido encontradas teses sobre $\mathrm{O}$ assunto, o número de publicações é baixo, fatos igualmente constatados por Alves et al. (2012, p. 313), que concluíram que

o levantamento bibliográfico levado a efeito demonstra a existência de poucos estudos publicados quanto ao papel dos psicólogos diante dos desastres, principalmente no tocante a países como o Brasil e a Espanha, sendo que no Brasil a situação ainda é bem mais precária. Isso caminha na contramão da tendência atual de aumento dos desastres naturais e da consequente necessidade de prestar auxílio multiprofissional às pessoas e comunidades afetadas.

Com relação ao método utilizado nos estudos, no que diz respeito aos artigos, três deles são relatos de experiências de atendimentos, sendo que o primeiro versa sobre o atendimento de amigos e familiares das vítimas de acidentes aéreos (Franco, 2005); o segundo, sobre o acolhimento das vítimas do incêndio na Boate Kiss (Cabral \& Simoni, 2013); e o último, sobre uma intervenção depois dos 
desmoronamentos na região serrana do Rio de Janeiro (Weintraub, Noal, Vicente \& Knobloch, 2014). Foi também encontrado um estudo de caso (Silva et al., 2013) que, do mesmo modo, trata do atendimento aos familiares e vítimas do incêndio na Boate Kiss, em Santa Maria, e ainda uma pesquisa empírica que entrevistou psicólogos com prática no atendimento em emergências e desastres (Trindade \& Serpa, 2013). Os outros seis estudos (Albuquerque $\&$ Zacarias, 2016; Alves et al., 2012; Guimarães, Guimarães, Neves \& Cistia, 2007; Meller, 2015; Melo \& Santos, 2011; Sá, Werlang \& Paranhos, 2008) são revisões de literatura.

No que se refere às duas monografias (Franco, 2013; Silva, 2013), Franco (2013), a partir da sua experiência profissional em clínica psicanalítica e saúde pública, realizou uma revisão crítica da literatura, utilizando o caso do incêndio na Boate Kiss. A finalidade de seu estudo foi explorar a especificidade das situações de emergências e desastres como campo de intervenção da saúde mental. A autora cita algumas situações específicas que podem solicitar o trabalho do psicólogo de modo diferente do convencional, como acompanhar um familiar no reconhecimento de corpos. Ela argumenta que, nesses casos, "há um desamparo na linguagem que convoca o psicólogo a um amparo corporal. Às vezes, resta ao psicólogo oferecer um gesto quando não há fala possível, onde as palavras aparecem mais como voz do que como sentido: uma presença silenciosa" (Franco, 2013, p. 19). A autora ainda salienta que em uma situação de desastre o psicólogo não pode afirmar à pessoa em crise que "tudo vai melhorar", porém, levando em consideração que a crise é temporária, o profissional poderia dizer frases como "do jeito que está não vai ficar".

Já Silva (2013), por meio de uma revisão bibliográfica, elencou cinco conceitos da Psicologia Humanista que se relacionam diretamente com a atuação do psicólogo nas emergências e desastres: (i) compreensão do ser humano como um todo; (ii) noção de experiência; (iii) tendência atualizante; (iv) empatia; e (v) uma concepção positiva do ser humano. A pesquisa visou entender qual seria o papel do psicólogo humanista no auxílio de pessoas que vivenciaram desastres. O psicólogo começaria a atuar logo em seguida aos primeiros socorros físicos. É por meio da escuta empática que a vítima conseguirá comunicar suas demandas emocionais imediatas e se sentirá compreendida. Para a autora, não é o contexto, mas a percepção particular construída por meio de sua história pessoal, experiências e conflitos, que ditará a forma como cada um viverá uma situação de desastre.

No que concerne à dissertação, Cogo (2010) realizou um estudo a respeito da atuação dos psicólogos em situações de emergência, sendo a única dissertação encontrada. Nela, a autora buscou compreender a experiência e o significado da atuação do psicólogo em emergências, a partir de um estudo de caso, que abordou o ponto de vista do profissional e evidenciou alguns dos temas importantes para a formação do psicólogo que tem por objetivo seguir nessa área, a saber: trauma, luto e Transtorno do Estresse Pós-Traumático (TEPT). A revisão de literatura de Alves et al. (2012) também encontrou apenas uma dissertação, entretanto, tal trabalho, intitulado $O$ 
impacto e as estratégias de "coping" de indivíduos em comunidades afetadas por desastres naturais (Krum, 2007), é diferente do encontrado na presente pesquisa (Cogo, 2010). Já a única tese citada no estudo destes autores, A Psicologia das emergências: um estudo sobre a angústia pública e o dramático cotidiano do trauma (Bruck, 2007), foi encontrada durante a busca bibliográfica deste estudo, porém não se enquadrou nos critérios de inclusão.

\section{A Intervenção nas Diferentes Fases de} Atuação

No que se refere à intervenção propriamente dita, foi possível observar, por meio da análise dos 14 trabalhos, que além de existirem diferentes fases possíveis para a atuação e inúmeras possibilidades de intervenção em cada uma delas, não existe um consenso entre as publicações acadêmicas sobre as formas de atuação nessas situações. Isso se deve ao fato de que em um desastre, ou em uma situação de risco, cada caso é único e tem suas próprias peculiaridades e, como consequência, o modo de intervir em cada contexto específico para auxiliar as vítimas, parentes, socorristas, entre outros, também será única. Assim, cada autor encontrado na presente revisão bibliográfica buscou construir as formas de intervenção propostas ao psicólogo nesses contextos, seja por meio de relatos de experiências de atuação em desastres, por meio de estudos de outros temas úteis para o psicólogo, tais como luto, por exemplo, seja recorrendo a entrevistas com psicólogos com experiência em atuação nos desastres, ou mesmo mediante revisões bibliográficas. Tal profusão de procedimentos aponta para a lacuna de um protocolo específico voltado para psicólogos que atuam na realidade brasileira e extrapola a diversidade de atuação fomentada pela variabilidade teórica da Psicologia como ciência e profissão. Também apontam claramente a invisibilização da gestão de riscos e da prevenção, tendo o seu foco no acontecimento do desastre e na posvenção.

Os manuais de orientações técnicas que abordam a atuação dos profissionais de saúde na gestão integral de riscos e desastres, de acordo com Silva et al. (2013), têm como finalidade unificar a atuação do psicólogo no acolhimento das vítimas antes, durante e depois do evento adverso, visando à empatia e buscando auxiliar o desenvolvimento de estratégias de enfrentamento (coping). $\mathrm{O}$ estudo também evidencia a necessidade de uma maior preparação técnica por parte dos profissionais de Psicologia para atuações futuras.

Os manuais mais citados nos trabalhos utilizados na presente revisão foram as orientações do grupo National Organization for Victims Assistance - Nova (Cogo, 2010; Franco, 2005;), National Child Traumatic Stress Network \& National Center for PTSD (Franco 2013; Silva et al., 2013) e as recomendações da OMS (Cogo, 2010; Franco, 2013; Meller, 2015). Os demais protocolos de agências internacionais citados foram Salud Mental para Víctimas de Desastres: Manual para Trabajadores (Melo \& Santos, 2011), Australian Red Cross InterAgency Standing Committee (IASC) e National Child Traumatic Stress Network - NCTSN (Meller, 2015) e Care (Franco, 2013). Nota-se 
que todos são protocolos e manuais internacionais, construídos para diversos contextos diferentes entre si, produzindo, consequentemente, divergências sobre os procedimentos nos trabalhos aqui pesquisados e desconectados do contexto brasileiro.

$\mathrm{Na}$ delimitação das fases de atuação do psicólogo, apesar de os autores utilizarem termos diferentes ao se referirem aos momentos de intervenção, estes podem ser resumidos em três: antes, durante e depois do desastre. Entre todas as propostas de caracterização das fases de atuação do psicólogo apresentadas nos artigos analisados, a proposta de Melo e Santos (2011) pareceu ser a mais didática. Os autores dividiram a atuação do psicólogo em quatro fases: a primeira seria o momento pré-desastre ou fase de prevenção; a segunda, durante o desastre, é a fase de emergência; o momento pós-desastre foi dividido em duas fases, a saber, a fase assistencial, que corresponde à terceira fase, e a de reconstrução, quarta fase. No presente trabalho, a análise das possíveis intervenções dos psicólogos na gestão integral de riscos e de desastres apresentados pelos artigos encontrados foi estruturada a partir de três momentos possíveis de intervenção: prédesastre, durante o desastre e pós-desastre.

\section{Pré-Desastre}

O momento pré-desastre, que pode também ser denominado como "fase de prevenção" (Melo \& Santos, 2011), corresponde à etapa na qual o psicólogo poderia realizar trabalhos com a população com o objetivo de modificar a percepção de risco de modo a diminui-los (Albuquerque \& Zacarias, 2016). Sobre a percepção de riscos, Favero et al. (2016, p. 72) contrapõem a afirmação de Albuquerque e Zacarias, salientando que "o mais importante nesse processo, não é a mudança da percepção de risco em si, mas a qualificação desta, ou seja, a ruptura com concepções naturalizadas sobre os riscos presentes no ambiente e a busca de uma melhor convivência ambiental".

O profissional da saúde mental também pode auxiliar na criação de redes de apoio para o enfrentamento de emergências (Albuquerque \& Zacarias, 2016) direcionando as ações basicamente para o treino de respostas e para a construção de planos de emergência (Alves et al., 2012). Trindade e Serpa (2013) e Alves et al. (2012) destacam a necessidade de empoderamento da comunidade, incluindo-a como polo ativo na prevenção, sendo o psicólogo parte da equipe interdisciplinar nos programas de políticas públicas, nos programas educativos sobre prevenção de desastres e nos cursos de capacitação. Em comparação com a revisão de Alves et al., as intervenções recomendadas pelos autores no que se refere ao pré-desastre foram as mesmas. Os autores destacam ainda que o psicólogo deve ter uma visão sistêmica da situação e que fóruns nacionais de discussão e debates sobre a gestão integral de riscos e desastres devem ser permanentes. Para Cogo (2010), o psicólogo pode ainda fazer parte da equipe de socorristas realizando um trabalho com a própria equipe, desde a preparação em capacitações, atuando em treinamentos e em programas de prevenção a acidentes de trabalho e síndrome de burnout, assim como auxiliando no campo educacional 
instruindo professores e alunos.

No que se refere aos instrumentos possíveis de serem aplicados na fase pré-desastre, Alves et al. (2012) encontraram os seguintes: medição da ansiedade (STAI), investigação do sofrimento emocional (SRQ-20), verificação da incidência de TEPT (TSQ), diagnóstico de TEPT (Composite International Diagnostic Interview), diagnóstico de outros sintomas psicológicos (Self-Reporting Questionnaire), escala de investigação de impacto de eventos estressores (IES), inventário de depressão de Beck (BDI), rastreamento de doenças mentais e ideação suicida (escala K6), instrumento de medição de estresse (General Health Questionnaire - GHQ-12) e questionário autoaplicável para levantamento de sintomas de trauma - The Kauai Reaction Inventory - KRI e Child Reaction Inventory.

\section{Durante o Desastre}

$\mathrm{Na}$ fase de emergência, a intervenção do psicólogo pode ser direta ou indireta. $\mathrm{Na}$ intervenção direta, o trabalho consiste em atuar diretamente na escuta, por meio das entrevistas de apoio, e como portador de informações que ajudem na orientação das pessoas em meio ao caos. Os psicólogos podem também intervir, indiretamente, no preparo psicológico dos profissionais que atuam diretamente nos desastres.

No que diz respeito às intervenções diretas, no momento do desastre, o psicólogo pode trabalhar com o objetivo de aumentar a capacidade de resiliência da comunidade e a criação de vínculos de ajuda mútua
(Albuquerque \& Zacarias, 2016), podendo fazer parte da equipe de socorristas auxiliando as vítimas e suas famílias (Cogo, 2010). Nesse momento, o trabalho pode consistir em instalar planos de manejo hospitalar para pessoas em vulnerabilidade, sendo sugerida a aplicação do debriefing psicológico em pessoas com propensão ao desenvolvimento de TEPT (Alves et al., 2012). Guimarães et al. (2007), realizaram uma revisão crítica de literatura, não sistemática, a respeito da técnica mais utilizada para intervenção psicológica a vítimas de desastres. Criada por Jeffrey Mitchell no ano de 1983, a técnica Critical Incident Stress Debriefing (CIST) foi desenvolvida para ser aplicada nas primeiras 24 a 72 horas depois de um evento traumático e consiste numa espécie de catarse na qual as vítimas de uma situação traumática expressam os sentimentos e emoções que vivenciaram durante essa experiência, visando ao restabelecimento emocional. Apesar de muito utilizado, alguns autores (Silva et al., 2013) colocam ressalvas no uso do debriefing psicológico, tendo em vista que este não tem sustentação empírica, podendo prejudicar ainda mais aqueles que se encontram em situação vulnerável, além de aumentar o risco de TEPT. Franco (2005) também assevera que não se deve estimular a pessoa a falar sobre o que aconteceu, porém, se ela quiser falar, não se deve fugir do assunto. Olhando sob essa perspectiva, é possível dizer que o debriefing pode ser considerado como uma forma de induzir a vítima a falar sobre o evento traumático, o que pode ser desconfortável e trazer consequências indesejáveis.

Meller (2015) elencou seis intervenções 
que devem nortear os primeiros cuidados psicológicos: (i) contato: demonstrar calma, de modo que o sujeito se sinta mais protegido; (ii) segurança: verificar se o sujeito ainda corre algum risco e, se necessário, retirá-lo do local além disso, faz-se importante encaminhá-lo para satisfazer suas necessidades fisiológicas, como alimentação e descanso, deixando-o mais confortável; (iii) estabilidade: fornecer informações relacionadas ao desastre (para algumas pessoas, isso pode ser importante), bem como estar disponível para ouvir aqueles que precisam falar; (iv) coleta de informações: indagar quais as necessidades e preocupações dos indivíduos, de forma a avaliar se a assistência está suprindo suas necessidades; (v) conexão com a rede social: identificar os familiares, amigos e pessoas próximas aos envolvidos a fim de fortalecer seu suporte primário; e (vi) informar: indicar os serviços de assistência e informar sobre habilidades de enfrentamento e resiliência.

Diferentemente, Sá et al. (2008) utilizaram como referência as fases de uma proposta de intervenção em crise para primeiros cuidados psicológicos de Moreno, Peñacoba, González-Gutiérrez e Ardoy (2003) e de Raffo (2005), as quais correspondem a: (i) estabelecer contato psicológico: escuta empática; (ii) Analisar o problema: considerando o passado imediato, presente e futuro imediato do evento traumático; (iii) Analisar possíveis soluções: observar quais as tentativas de enfrentamento do sujeito; (iv) assistir para executar ações concretas: auxiliar no gerenciamento da crise; e (v) seguimento para verificar o progresso: buscar apoio das redes sociais, acompanhar a recuperação dos indivíduos.

A meta principal da intervenção é auxiliar o indivíduo a desenvolver resiliência, isto é, a continuar vivendo de uma forma adaptada a partir de um novo contexto depois do desastre. De acordo com Mateu, Gil e García-Renedo (2009), fala-se de resiliência quando o indivíduo, na sua interação com situações penosas (pobreza, doença, desastre), é fortalecido e capacitado para enfrentar as adversidades.

Sobre a experiência em atendimentos em situações de risco e de desastres, foram encontrados três artigos (Cabral \& Simoni, 2013; Franco, 2005; Weintraub et al., 2014). Cabral e Simoni visaram relatar suas experiências no atendimento dos familiares das vítimas do incêndio na Boate Kiss. Especificamente sobre o trabalho realizado no dia da tragédia, as autoras enfatizam que os cuidados psicológicos consistiam em "acolher sem invadir, ofertar cuidado sem calar a dor, acompanhar reconhecendo o vazio e a solidão da perda" (Cabral \& Simoni, 2013, p. 8).

Franco (2005), por sua vez, realizou um estudo fundamentado na experiência de um grupo de psicólogos em atendimentos a vítimas de acidentes aéreos, visando a uma ação preventiva para situações de TEPT e de luto traumático, bem como desenvolver habilidades nos profissionais envolvidos com essa atividade, de maneira a terem uma atuação eficiente, com risco controlado para sua saúde mental. A autora chama atenção para o cuidado a ser tomado com pessoas enlutadas e traumatizadas. Em situações extremas, as pessoas em condição de fragilidade estão "desorganizadas, incoerentes, assustadas e paralisadas" (Franco, 2005, p. 179) e 
precisam ser acolhidas pacientemente. Desse modo, Franco (2005, p. 179) afirma que o objetivo da gestão de riscos e de desastres não é "[...] fazer com que a pessoa pare de sofrer rapidamente, pois isto seria um mecanismo de tamponamento de sua reação com graves consequências. Assim sendo, cuidamos para não evitar o assunto e não desviar a conversa do tema", apontando mais uma vez para o debriefing como uma técnica controversa.

Weintraub et al. (2014) realizaram um estudo a partir das suas experiências em situações de desastres em 2011, na região serrana do Rio de Janeiro. O principal aspecto ressaltado pelas autoras foi a importância da ação do psicólogo em uma rede articulada. Ao pensar a teoria e a prática, Weintraub et al. dividiram a intervenção em quatro pontos: (i) objetivo da intervenção: o restabelecimento das redes comunitárias e públicas de saúde mental, visto que o foco da ação era mais nas equipes dos serviços públicos do que na população atingida; (ii) tempo de duração: nesse caso específico, o tempo de atuação foi de 30 dias, pois as autoras não queriam correr o risco de deslegitimar as equipes de saúde locais; (iii) relevância: tal intervenção se mostrou importante, pois teve como intuito orientar as equipes de saúde locais sobre como atender as vítimas dos desastres; e (iv) impacto: este item foi analisado em dois aspectos, a saber, individual e comunitário, ambos devem ter seus contextos considerados. Dessa forma, consideram que a intervenção do psicólogo em desastres deve, em primeiro lugar, estar de acordo com o contexto de cada comunidade específica. E ainda, o manejo da crise deve ter início nesse reconhecimento, no qual o profissional da Psicologia tomará conhecimento dos equipamentos da rede socioassistencial envolvidos com a comunidade, bem como das demandas geradas pelo desastre. Além disso, as autoras salientam que, em casos de emergências, suprir as necessidades básicas e fisiológicas humanas são também ações de saúde mental, e que o psicólogo deve atender e encaminhar as vítimas para que tenham o mínimo de conforto. O psicólogo também assume um papel de articulador entre os vizinhos, as famílias e a comunidade, pois estes são a base do fortalecimento e da recuperação.

A respeito do momento do desastre, Alves et al. (2012) encontraram estudos que versam basicamente sobre a importância da detecção de pessoas vulneráveis ao desenvolvimento do TEPT por meio da aplicação do debriefing psicológico. Diversamente de Alves et al., a presente revisão da literatura encontrou publicações acadêmicas que abordam especificamente sobre como intervir nos primeiros cuidados psicológicos (Meller, 2015; Sá et al., 2008), bem como relatos de experiências de psicólogos que atuaram em situações de emergências e desastres e escreveram sobre as peculiaridades de cada situação vivenciada (Cabral \& Simoni, 2013; Franco, 2005; Weintraub et al., 2014).

\section{Pós-desastre}

Assim como nos achados de Alves et al. (2012), o momento pós-desastre foi o mais discutido nas publicações acadêmicas da presente pesquisa, apontando para o fato de que 
talvez ainda estejamos muito vinculados à ideia de que a atuação do psicólogo está mais vinculada ao tratamento do que à prevenção. Alves et al. apontam para a relevância da intervenção do psicólogo no sentido de diminuir as consequências para a saúde mental das vítimas de desastres e de seus familiares. As principais ações sugeridas foram a avaliação do sofrimento psíquico das vítimas com enfoque na prevenção do desenvolvimento do TEPT (assim como no momento do desastre) e a realização de estudos que avaliem o desempenho dos psicólogos nas intervenções realizadas de modo a subsidiar ações futuras. Apesar de o debriefing ser citado por Alves et al. como uma técnica para intervenção durante o desastre, Guimarães et al. (2007) o citam como técnica de intervenção depois do desastre. Salienta-se que os trabalhos citados por Alves et al. no pós-desastre são, em sua maioria, estrangeiros, e isso pode significar que talvez os estudos nacionais estejam mais preocupados com a atuação nos momentos pré e durante o desastre em comparação aos internacionais. De qualquer modo, é preocupante que a literatura nacional esteja disseminando a ideia de que o debriefing deva ser utilizado em situações de emergências e desastres, quando os PCP têm sido indicados internacionalmente como seu substitutivo $\mathrm{e}$ primeira opção de intervenção, por órgãos tais como o Comitê de Interagências (IASC) e o projeto Sphere, assim como o Programa de Ação Global para Superação das Lacunas em Saúde Mental da OMS - mhGAP (OMS, 2015).

A fase assistencial refere-se à ajuda humanitária propriamente dita e envolve ações tais como suprimento de elementos básicos de sobrevivência (água, alimentos, roupas), encaminhamento de famílias desalojadas, entre outros. Aqui, o conhecimento técnico do psicólogo pode ser de grande auxílio, pois abrange questões pessoais importantes, como mulheres grávidas, famílias, idosos, crianças, etc. (Melo \& Santos, 2011). O psicólogo pode ainda trabalhar com os assistentes sociais nos hospitais na recepção de feridos e seus familiares e realizar ações de modo a facilitar a volta ao trabalho (Cogo, 2010).

Já na fase de reconstrução, o psicólogo pode atuar nas elaborações de políticas públicas, observando as demandas específicas de cada comunidade (Melo \& Santos, 2011), por meio de ações que se constituem no levantamento dos impactos psicológicos de todas as pessoas envolvidas, tanto as vítimas como o pessoal da equipe de socorro e avaliação dos eventos, de modo a construir uma atuação mais eficaz no futuro (Alves et al., 2012; Albuquerque \& Zacarias, 2016). Ressalta-se que a elaboração de políticas públicas é proposta tanto no momento pré-desastre quanto no momento pós-desastre. Pode-se pensar em um movimento cíclico, tendo em vista que no pós-desastre as experiências de atendimento durante o desastre são utilizadas como referência para a criação das políticas públicas.

\section{Considerações Finais}

Os dados da presente pesquisa nos mostram que o Brasil é ainda incipiente nos estudos sobre a Psicologia da gestão integral de riscos e de desastres, confirmando a precariedade da produção encontrada na 
literatura (Alves et al., 2012). Apesar das tragédias ocorrerem com certa frequência, as publicações acadêmicas a respeito do tema são em sua maioria artigos de revisão ou de cunho teórico. Outro aspecto relevante é o fato de que nesta revisão da literatura encontrou-se apenas uma dissertação e nenhuma tese que discuta a intervenção do psicólogo na gestão de riscos e desastres, sendo o artigo mais antigo datado do ano de 2005. Diante disso, torna-se necessário que sejam realizadas novas pesquisas para a consolidação desses dados e elaboração de protocolos para a ação dos psicólogos, não apenas no pós-desastre, mas também nas fases anteriores, especialmente no que diz respeito à gestão de riscos que envolvem ações de prevenção em saúde mental.

Um dos aspectos que pode ser ressaltado diz respeito ao impacto dos descritores utilizados na presente investigação, indicando que é necessária uma ampliação destes nas próximas pesquisas, dificuldade também encontrada por Alves et al. (2012). Provavelmente, o fato de a área ainda estar em consolidação provoca dificuldades na catalogação dos trabalhos por palavras-chave na base de dados. Temos por exemplo que, de acordo com a OMS (2015), o termo correto a ser utilizado é primeiros "cuidados" psicológicos, embora haja na literatura com frequência o termo "primeiros socorros psicológicos", utilizado nas buscas do presente trabalho, podendo, portanto, ser uma limitação para os resultados encontrados. Ainda com relação aos descritores, as buscas realizadas podem não ter localizado a totalidade das publicações acadêmicas devido ao fato de que algumas revistas não aceitam palavras-chave que não estejam incluídas no dicionário da BVS-Psi.

Outro problema encontrado na pesquisa, e que tem impacto no mapeamento realizado, diz respeito ao fato de que não existe um consenso sobre a forma de intervenção psicológica entre os autores. Quanto às orientações para a atuação do psicólogo em cada momento, o que se pode concluir é a urgência da inclusão desse profissional nas diferentes equipes de atuação nos desastres, incluindo aqui a Defesa Civil. O psicólogo pode atuar não apenas no pós-desastre, mas também na promoção de capacitações e palestras para a conscientização das comunidades, assim como na formulação de políticas públicas, o que infelizmente ainda não faz parte da realidade brasileira, atuando assim na gestão de riscos e ampliando a concepção do que é emergência e desastre, bem como o que significa intervir nessas situações.

Ademais, uma atenção especial deve ser dada ao fato de os autores brasileiros insistirem na técnica do debriefing psicológico. No ano de 2009 o debriefing foi substituído pelos PCP, depois de estudos internacionais apontarem a técnica como ineficaz (OMS, 2015). Segundo a OMS (2015, p. 3), os PCP consistem em "uma resposta humana e de apoio às pessoas em situação de sofrimento e com necessidade de apoio". Os PCP visam

oferecer apoio e cuidado prático não invasivos; avaliar necessidades e preocupações; ajudar as pessoas a suprir suas necessidades básicas (por exemplo, alimentação, água e informação); escutar as pessoas, sem pressioná-las a falar; confortar as pessoas e ajudá-las a se sentirem calmas; ajudar as pessoas na 
busca de informações, serviços e suportes sociais; proteger as pessoas de danos adicionais. (OMS, 2015, p. 3)

Como sugestão para futuros estudos, destaca-se a importância do desenvolvimento de projetos mais amplos, tais como oficinas de prevenção dos desastres em comunidades vulneráveis e ações de capacitação em PCP para profissionais que atuam nessas ocasiões, bem como estudos mais específicos, interessados no sofrimento das vítimas, que busquem formas de auxiliar na ressignificação da sua dor e recuperar, na medida do possível, a sua saúde mental. Além disso, é necessário que os cursos de graduação em Psicologia abordem o tema da atuação do psicólogo na gestão integral de riscos e de desastres, posto que o Código de Ética Profissional do Psicólogo - CEPP (2005) dita que o profissional em saúde mental tem o dever de auxiliar em situações de calamidades públicas e emergências, mas, também, como aponta no art. $1^{\circ}$, alínea b, só pode assumir responsabilidades profissionais, para as quais esteja "capacitado pessoal, teórica e tecnicamente" (CEPP, 2005, p. 8).

A área da Psicologia na gestão integral de riscos e de desastres é muito ampla e abrange temáticas diversas e relevantes, além da apresentada aqui. Entre tais temáticas, o autocuidado do psicólogo e de seus colegas de equipe é um tema que merece destaque, pois em situações extremas é essencial que o psicólogo tenha condições de desempenhar a sua função da melhor forma, visto que também estarão sob tensão e submetidos a um alto grau de estresse durante os atendimentos.

Por fim, destacamos o papel do psicólogo nas fases anteriores ao desastre, pois, como em Alves et al. (2012), neste trabalho encontramos maior ênfase nas fases pósdesastre. O psicólogo pode e deve atuar com prevenção e gestão de riscos, desenvolvendo trabalhos tais como a conscientização da população, o treinamento dos profissionais responsáveis pelos primeiros socorros e o fortalecimento do vínculo da população com os equipamentos da rede de serviços local, ações imprescindíveis para prevenir desastres com grandes danos, ou até mesmo a sua ocorrência. Sabe-se que os países que investem nesse preparo contam com um número muito menor de vítimas quando ocorrem acidentes.

\section{Referências ${ }^{4}$}

*Albuquerque, B. S., \& Zacarias, G. M. (2016). A Psicologia como aliada à gestão de risco em desastres. Revista Ordem Pública e Defesa Social, 9(1), 109-120. Recuperado em 12 dezembro, 2017, de https://rop.emnuvens.com.br/rop/article /view/113.

*Alves, R. B., Lacerda, M. A. C., \& Legal, E. J. (2012). A atuação do psicólogo diante dos desastres naturais: uma revisão. Psicologia em Estudo, 17(2), 307-315. Doi: 10.1590/S1413-73722012000200014.

Britton, N. R. (1986). Developing an Understanding of Disaster. Journal of Sociology, 22(2), 254-271. Recuperado em 21 julho, 2018, de http://journals.sagepub.com/doi/10.117 7/144078338602200206.

Bruck, N. R. V. (2007). A Psicologia das emergências: um estudo sobre angústia pública e o dramático cotidiano do trauma. Tese de doutorado, Pontifícia Universidade Católica do Rio Grande do Sul, Porto Alegre, Brasil.

\footnotetext{
4 Referências precedidas por asterisco são os artigos encontrados na revisão.
} 
*Cabral, V. K., \& Simoni, A. C. R. (2013). Fazendo a gestão no olho do furacão. Entrelinhas, 62, 8-9. Recuperado em 5 dezembro, 2017, de http://www.crprs.org.br/upload/edicao/ arquivo57.pdf.

Centro Universitário de Estudos e Pesquisas sobre Desastres, Universidade Federal de Santa Catarina. (2013). Atlas brasileiro de desastres naturais: 1991 a 2012 (2a ed.). Florianópolis: Ceped/UFSC. Recuperado em 5 dezembro, 2017, de http://www.ceped.ufsc.br/wpcontent/uploads/2012/01/AMAZONA S_mioloWEB.pdf.

Centro Universitário de Estudos e Pesquisas sobre Desastres, Universidade Federal de Santa Catarina, Secretaria Nacional de Defesa Civil, Ministério da Integração Nacional. (2010). Gestão de riscos e de desastres: contribuições da psicologia. Florianópolis: Autor. Recuperado em 5, dezembro, 2017, de http://www.integracao.gov.br/c/docume nt_library/get_file?uuid=8fa26fe8-d31a4531-92ca-

346e6c69867f\&groupId=10157.

*Cogo, A. S. (2010). O psicólogo com atuação em emergências: experiência e significado. Dissertação de mestrado, Pontifícia Universidade Católica de São Paulo, São Paulo, Brasil.

Conselho Federal de Psicologia. (2005). Código de Ética do Profissional Psicólogo. Brasília: Autor. Recuperado em 5 dezembro, 2017, de https:// site.cfp.org.br/wpcontent/uploads/2012/07/codigo-deetica-psicologia.pdf.

Conselho Federal de Psicologia. (2013). Nota técnica sobre atuação de psicóloga(o)s em situações de emergências e desastres, relacionadas com a política de defesa civil. Brasília: Autor. Recuperado em 5 dezembro, 2017, de http://www.crpsp.org.br/portal/orientac ao/recomendacoes/nota-tecnica-cfpemergencias.pdf.

Conselho Federal de Psicologia. (2016). Nota técnica sobre atuação da psicologia na gestão integral de riscos e de desastres, relacionadas com a política de proteção e defesa civil. Brasília:
Autor. Recuperado em 5 dezembro, 2017, de http://www.crpsp.org.br/emergencias/p df/Nota-Tecnica-Psicologia-Gestao-deRiscos.pdf.

Favero, E., Sarriera, J. C., Trindade, M. C., \& Galli, F. (2013). In J. F. Leite \& M. Dimenstein (Orgs.). Psicologia \& Contextos Rurais (pp. 303-332). Natal: EDUFRN.

Favero, E., Sarriera, J. C., \& Trindade, M. C. (2014). O desastre na perspectiva sociológica e psicológica. Psicologia em Estudo, 19(2), 201-209. Doi: 10.1590/1413-737221560003.

Favero, E., Trindade, M. C., Passuello, A., Pauletti, C., Foresti, A. J., Sarriera, J. C., \& Filho, L. C. P. S. (2016). Percepção de risco ambiental: uma análise a partir de anotações de campo. Revista Interamericana de Psicologia, 50(1), 64-74. Recuperado em 5 dezembro, 2017, https://journal.sipsych.org/index.php/IJ $\mathrm{P} /$ article/view/42.

Franco, M. H. P. (2005). Atendimento psicológico para emergências em aviação: a teoria revista na prática. Estudos de Psicologia, 10(2), 177-180. Doi: 10.1590/S1413-294X2005000200003.

*Franco, M. H. P. (2015). Intervenção psicológica em emergências: fundamentos para a prática. São Paulo: Summus.

*Franco, M. H. C. (2013). Saúde mental em emergências e desastres: contribuições à prática do psicólogo. Monografia de graduação em Psicologia, Universidade Federal do Rio Grande do Sul, Porto Alegre.

Fritz, C. E. (1961). Disaster. In R. K. Merton \& R. A. Nisbet (Org.). Contemporary Social Problems (pp. 651-694). New York: Harcourt, Brace and World.

Garcia-Renedo, M., Beltrán, J. M. G., \& Valero, M. V. (2007). Psicología y desastres: aspectos psicosociales. Castelló de la Plana: Publicacions de la Universitat Jaume I.

*Guimarães, L. A. M., Guimarães, P. M., Neves, S. N. H., \& Cistia, J. M. D. (2007). A técnica de debriefing psicológico em 
acidentes e desastres. Mudanças Psicologia da Saúde, 15(1), 1-12. Doi:

10.15603/2176-1019/mud.v15n1p1-12.

Krum, F. M. B. (2007). O impacto e as estratégias de "coping" de individuos em comunidades afetadas por desastres naturais. Dissertação de mestrado, Universidade Federal do Rio Grande do Sul, Porto Alegre, Brasil.

Lopes, D. C., \& Filho, O. S. (2017). O psicólogo na redução dos riscos e desastres: teoria e prática. São Paulo: Hogrefe.

Mateu, R., Gil, J. M., \& García-Renedo, M. (2009). ¿Hacia una escuela resiliente? Un estudio a través del profesorado de Educación Infantil y Primaria. Dissertação de mestrado, Universitat Jaume I, Castelló de la Plana, Espanha.

McFarlane, A. C., \& Norris, F. H. (2006). Definitions and Concepts in Disaster Research. In F. H. Norris, S. Galea, M. J. Friedman \& P. J. Watson (Orgs.). Methods for Disaster Mental Health Research (pp. 319). New York: Guilford Publications.

*Meller, V. (2015). Primeiros auxílios psicológicos para indivíduos envolvidos em situações emergenciais e desastres. Diaphora, 15(1), 55-59. Recuperado em 5 dezembro, 2017, de http://www.sprgs.org.br/diaphora/ojs/in dex.php/diaphora/article/view/97/95.

*Melo, C. A., \& Santos, F. A. (2011). As contribuições da Psicologia nas emergências e desastres. Psicólogo Informação, 15(15), 169-181. Recuperado em 5 dezembro, 2017, de https://www.metodista.br/revistas/revist as-

$\mathrm{ims} /$ index.php/PINFOR/article/view/3 $177 / 3045$.

Moher, D., Liverati, A., Tetzlaff, J., \& Altman, D. G. (2015). Principais itens para relatar revisões sistemáticas e meta-análises: a recomendação Prisma. Epidemiologia e Serviços de Saúde, 24(2), 335-342. Doi: 10.5123/S1679-49742015000200017.

Moreno, R. R., Peñacoba, C. P., GonzálezGutiérrez, J. L., \& Ardoy, J. C. (2003). Intervención psicológica en situaciones de crisisy emergencias. Madrid: Dykinson.

Organização Pan-Americana da Saúde. Ministério da Saúde. (2014). Desastres Naturais e Saúde no Brasil (Série

Desenvolvimento Sustentável e Saúde, 2). Brasília: Autor. Recuperado em 12 dezembro, 2017, de http://www.paho.org/bra/index.php?opt ion $=$ com_docman\&view $=$ download\&alia $\mathrm{s}=1513$-desastres-naturais-e-saude-nobrasil-3\&category_slug=saudereve-eambiente-707\&Itemid=965.

Organização Mundial da Saúde, War Trauma Foundation, Visão Global internacional. (2015). Primeiros Cuidados Psicológicos: guia para trabalhadores de campo. Genebra: OMS. Recuperado em 12 dezembro, 2017, de http://www.paho.org/bra.../index.php?o ption $=$ com_docman\&view $=$ download \&c ategory_slug=prevencaorevenção-e-contdoencas-e-desenv-sustentavel071\&alias $=1517$-primeiros-cuidadospsicologicos-um-guia-para-trabalhadorescampo-7\&Itemid $=965$.

Quarantelli, E. L. (1985). What is Disaster? The Need for Clarification in Definition and Conceptualization in Research. In B. Sowder (Org.). Disasters and Mental Health Selected Contemporary Perspectives (pp. 41-73). Washington, D.C.: Government Printing Office.

Raffo, S. L. (2005). Intervención en crisis. [Anotações de uso exclusivo para docência]. Departamento de Psiquiatría y Salud - Campus Sur. Universidad de Chile. Recuperado em 12 dezembro, 2017, de https://www.ucursos.cl/medicina/2009/1/MPSIQUI5/ 2/material_docente/bajar?id_material $=20$ 7751 .

*Sá, S. D., Werlang, B. S. G., \& Paranhos, M. E. (2008). Intervenção em crise. Revista Brasileira de Terapias Cognitivas, 4(1), 1-10. Recuperado em 5 dezembro, de 2017, de http://pepsic.bvsalud.org/scielo.php?scri $\mathrm{pt}=$ sci_arttext\&pid $=\mathrm{S} 1808$ 56872008000100008 .

Secretaria Nacional de Defesa Civil, Departamento de Prevenção e Preparação, Ministério da Integração Nacional. (2017). 
Módulo de formação: noções básicas em proteção e defesa civil e em gestão de riscos: livro base. Brasília: Autor. Recuperado em 12 dezembro, 2017, de http://mi.gov.br/documents/3958478/0 /I+-+Gestao+de+Risco++Livro+Base.pdf/7f00f4ac-14ba-4813b3d3-561a703d62a7.

*Silva, V. B. (2013). A Psicologia nas situações de emergências e desastres: uma reflexão humanista. Monografia de graduação em Psicologia, Universidade Estadual da Paraíba, Campina Grande, Brasil.

*Silva, T. L. G., Mello, P. G., Silveira, K. A. L., Wolffenbuttel, L., Lobo, B. O. M., Bicca, C. H. M., Grassi-Oliveira, R., \& Kristensen, C. H. (2013). Primeiros Socorros Psicológicos: relato de intervenção em crise em Santa Maria. Revista Brasileira de Psicoterapia, 15(1), 93104. Recuperado em 5 dezembro, 2017, de http://www.rbp.celg.org.br/detalhe_artig o.asp?id $=113$.

*Trindade, M. C., \& Serpa, M. G. (2013). O papel dos psicólogos em situações de emergências e desastres. Estudos e Pesquisas em Psicologia, 13(1), 279-297. Recuperado em 5 dezembro, 2017, de http://pepsic.bvsalud.org/scielo.php?scri $\mathrm{pt}=$ sci_arttext\&pid $=\mathrm{S} 1808$ 42812013000100017.

*Weintraub, A. C. A. M., Noal, D. S., Vicente, L. N., \& Knobloch, F. (2014). Atuação do psicólogo em situações de desastre: reflexões a partir da práxis. Comunicação Saúde Educação, 19, 287-298. Doi: 10.1590/1807-57622014.0564.

Recebido em: 14/12/2017

Aprovado em: 30/7/2018 